\title{
The Addition of High Intensity Interval Training Reduces Cardiovascular Disease Risk Factors and Enhances Strength in Active, Healthy Adults
}

\author{
Jinger S. Gottschall ${ }^{1}$, Christopher M. Bopp ${ }^{1}$, Bryce Hastings ${ }^{2}$ \\ ${ }^{1}$ The Pennsylvania State University, University Park, USA \\ ${ }^{2}$ Les Mills International, Auckland City, New Zealand \\ Email: jinger@psu.edu
}

Received 17 March 2014; revised 18 April 2014; accepted 8 May 2014

Copyright (C) 2014 by authors and Scientific Research Publishing Inc. This work is licensed under the Creative Commons Attribution International License (CC BY). http://creativecommons.org/licenses/by/4.0/

(c) (i) Open Access

\section{Abstract}

The guidelines for cardiovascular exercise intensity are not clear with respect to the optimal training protocols to reduce cardiovascular disease risk (CVD) as well as to enhance strength. At this time, it is impossible to deduce the independent effects of vigorous-intensity exercise from past studies as the volume of either physical activity duration or energy expended differed in comparison to moderate-intensity exercise. Our goal was to augment the literature focused on the potential benefits of high intensity training with novel methodology that includes trained adults in a group fitness environment. Vigorous intensity training may be a critical component of the physical activity prescription if active adults demonstrate decreases in CVD risk factors and increases in strength. We hypothesize that a 6-week high intensity group fitness intervention will produce beneficial effects on CVD risk factors by reducing body mass, total cholesterol, LDL-C, triglyceride concentration, and by enhancing lean body mass percentage, HDL-C, glucose tolerance, and maximal oxygen consumption as well as both upper and lower body strength. Eighty-four healthy adults, 50 women and 34 men, completed the 6-week intervention. Participants were randomly assigned to one of two groups; the first was a high intensity interval-training (GRIT) program, while the second was a moderate intensity-training (FIT) program. All participants completed 5 hours of fitness classes per week. We collected musculoskeletal and physiological data at the start and finish of the study. In support of our hypotheses, the GRIT group significantly reduced body mass, triglyceride concentration and enhanced lean body percentage, glucose tolerance, and maximal oxygen consumption in addition to all of the strength measures. These results illustrate a novel finding that individuals who already participate in the recommended physical activity guidelines can improve their health, fitness and strength with the addition of 2, 30-minute sessions of high intensity interval training per week. 


\section{Keywords}

\section{Physical Fitness Guidelines, Group Exercise, Cholesterol, Triglycerides, Body Mass}

\section{Introduction}

In the past 5 years, 1 of every 3 deaths in the United States was attributed to cardiovascular disease (CVD), which equates to 2200 Americans each day or 1 death every 30 seconds [1]. A sedentary lifestyle is one of two primary behavioral factors associated with the advancement of CVD. Despite these alarming statistics, 93\% of adult Americans do not satisfy the current physical activity guidelines, $60 \%$ are not regularly physically active and $25 \%$ are not active at all [2]. It is critical that research scientists, medical practitioners, and funding agencies strive to obtain easy to follow exercise prescriptions to reverse these trends.

Unfortunately, due to lack of data, the present recommendation for cardiovascular exercise is not straightforward. Recently, the American College of Sports Medicine published a position stand to provide guidance to professionals who prescribe exercise programs with a focus on how to reduce CVD risk factors [3]. They suggest that adults engage in cardiovascular exercise of moderate-intensity for 30 - 60 minutes at least 5 days per week, vigorous-intensity for 20 - 60 minutes at least 3 days per week, or a combination of these moderate- and vigorous-intensity regimens 3 - 5 days per week. The position stand authors admit that these guidelines for cardiovascular exercise are not clear because there is an obvious gap in the current literature with respect to the role of exercise intensity in reducing CVD risk factors [4]. Thus, at this time, it is impossible to deduce the independent effects of vigorous-intensity exercise from past studies as the volume of either physical activity duration or energy expended differed in comparison to moderate-intensity exercise [5]-[9].

Multiple groups have published promising results that vigorous-intensity training results in greater improvements in individual cardiovascular disease risk factors than moderate-intensity in adults who were not active before the beginning of the intervention. For instance, $\mathrm{VO}_{2}$ max increased and plasma lipids decreased after high intensity interval training compared to low intensity aerobic exercise [5]-[9]. In addition, DiPietro et al. [11] reported significant improvements in glucose utilization in sedentary older men and women who engaged in physical activity equal to $80 \%$ of maximal oxygen consumption but not in the group who engaged in activity equal to $65 \%$ maximal oxygen consumption.

These classic high intensity interval training studies, as well as thorough subsequent studies, have provided overwhelming evidence that vigorous intensity training significantly lowers CVD risk factors in sedentary adults [5]-[11]. The investigators utilized controlled protocols on either a treadmill or cycle ergometer in a laboratory setting. Our goal was to augment the literature focused on the potential benefits of high intensity training with novel methodology that includes trained adults in a group fitness environment. Vigorous intensity training may be a critical component of the physical activity prescription if active adults demonstrate decreases in CVD risk factors. We hypothesize that a 6-week high intensity group fitness intervention will produce beneficial effects on CVD risk factors by reducing body fat mass, total cholesterol, LDL-C, triglyceride concentration, and by enhancing lean body mass percentage, HDL-C, glucose tolerance and maximal oxygen consumption as well as both upper and lower body strength.

\section{Methods}

\subsection{Participants}

Eighty-four healthy adults (age $38.8 \pm 11.8$ yrs, height $1.7 \pm 0.1 \mathrm{~m}$ ), 50 women and 34 men, completed the 6-week intervention (Table 1). All of the participants gave written informed consent that followed the guidelines of The Pennsylvania State University Human Research Committee. The initial inclusion criteria for the study was for the participant to be between the age of 25 and 65, completing a minimum of 2 cardiorespiratory group fitness classes (Les Mills BODYATTACK ${ }^{\mathrm{TM}}$, BODYCOMBAT ${ }^{\mathrm{TM}}$, BODYSTEP ${ }^{\mathrm{TM}}$, RPM ${ }^{\mathrm{TM}}$ ) and 1 strength class (Les Mills BODYPUMPТМ) per week during the previous 6 months, and available during the 6 week period without more than four days of travel in a single week. We excluded potential participants from the study, in accordance to the ACSM health screening for physical activity, if he or she had any of the following conditions: 
Table 1. Participant $(n=84)$ characteristics at initial measurement; mean (standard deviation).

\begin{tabular}{ccc}
\hline & FIT & GRIT \\
\hline Age (yr) & $39.3(11.7)$ & $38.3(10.6)$ \\
Height (cm) & $169.8(10.0)$ & $170.0(11.1)$ \\
\hline
\end{tabular}

chest discomfort with exertion, unreasonable shortness of breath, symptoms of dizziness, fainting or blackouts, heart medication, asthma or other lung disease, burning or cramping sensations in lower legs with minimal physical activity, joint problems that limit physical activity, prescription medications, pregnancy, diabetes, smoking, blood pressure greater than 140/90 mmHg, or total blood cholesterol greater than $220 \mathrm{mg} \cdot \mathrm{dL}^{-1}$.

\subsection{Measurements}

We collected musculoskeletal and physiological data at the start (Week 0) and finish (Week 7) of the study. Blood was drawn from a finger prick after a 48-hour alcohol and a 12-hour food as well as exercise fast. The samples were assayed for total cholesterol, LDL-C, HDL-C, triglycerides, and glucose. Body composition was calculated using the US Navy Method with circumference measurements of the waist, neck, and hips (women only). We also conducted a submaximal oxygen consumption test using the Queens College Step Test. Finally we completed upper body, back, and leg strength tests with a maximal number of push-ups in one-minute test in addition to deadlift and squat isometric dynamometer tests.

\subsection{Physical Fitness and Nutrition Protocol}

Participants were randomly assigned to one of two groups; the first was a high intensity interval-training (GRIT) program, while the second was a moderate intensity-training (FIT) program. All participants completed 5 hours of fitness classes per week; GRIT = 2 high intensity classes (LES MILLS GRIT ${ }^{\mathrm{TM}}$ ), 2 cardiorespiratory classes, and 2 strength classes and FIT $=3$ cardiorespiratory classes and 2 strength classes. The participants completed Les Mills ${ }^{\mathrm{TM}}$ instructed group fitness classes at The North Club in State College, Pennsylvania. Each class was offered nearly every day of the week with classes beginning at 5:45 am until 6:45 pm allowing each participant to create an individual exercise schedule on a weekly basis. The participants independently selected their cardiorespiratory classes on a weekly basis from BODYATTACK ${ }^{\mathrm{TM}}$ (sports-inspired intervals), BODYCOMBATтM (mixed martial arts), BODYSTEPTM (height-adjustable bench) and RPM ${ }^{\mathrm{TM}}$ (indoor cycling). The muscular strength class, BODYPUMPTM incorporates a barbell as well as free weights. The high intensity interval training class, LES MILLS GRIT ${ }^{\mathrm{TM}}$ series is a 30-minute strength and plyometric workout. Finally, the participants were asked not to alter their daily diet habits and recorded their nutritional intake 2 days per week to determine if diet was altered, particularly for the participants that altered their physical fitness routine by adding vigorous intensity.

\subsection{Statistics}

We evaluated between- and within-group data with a two-factor mixed ANOVA design and with a one-way, repeated measures ANOVA. When a significant interaction was detected, data were subsequently analyzed using a Newman-Keuls post hoc test with significance defined at $\mathrm{p}<0.05$. Data are presented at mean \pm standard deviation.

\section{Results}

In support of our hypotheses, the high intensity interval, group fitness training program significantly reduced body fat mass, triglyceride concentration and enhanced lean body percentage, glucose tolerance, and maximal oxygen consumption as well as all of the strength measures. However, there were no statistical differences in any of the cholesterol measurements. To add, the compliance rate was $97.7 \%$ for the GRIT group and $98.7 \%$ for the FIT group, illustrating that there were less than 30 workouts missed out of the 2772 prescribed sessions.

Body composition was significantly less for the GRIT group after the participants completed the high intensity interval classes (Figure 1). Mean body mass decreased by a statistically significant $1.3 \mathrm{~kg}$ for the GRIT par- 


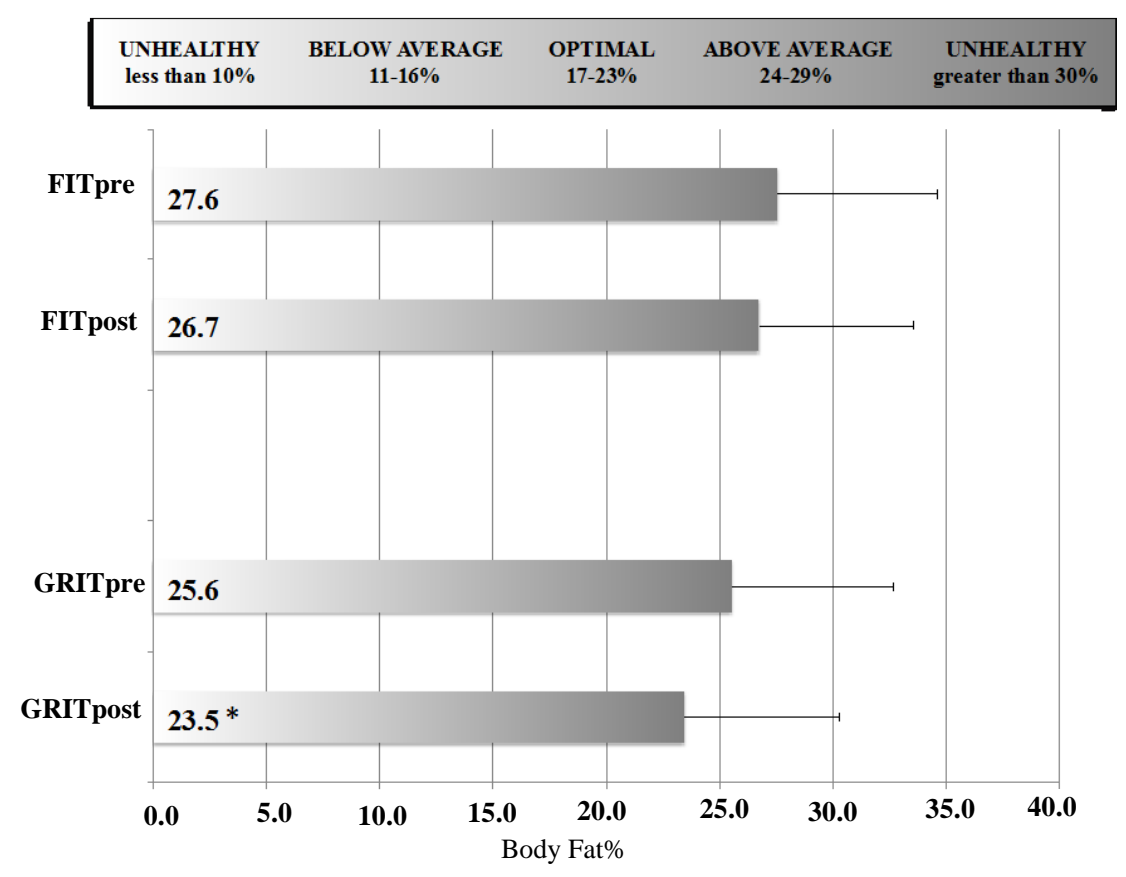

Figure 1. Mean \pm standard deviation for the initial and final measurements of body fat percent calculations for the FIT and GRIT groups. The asterisk indicates a significant difference $(p<0.05)$ between the initial and final measurements. The bar at the top of the figure illustrates the category and range for each variable.

ticipants $(\mathrm{p}<0.05)$ and an insignificant $0.4 \mathrm{~kg}$ for the FIT participants. Similarly, the GRIT group demonstrated a reduction in body fat of $2.0 \%(\mathrm{p}=0.01)$ while there was no significant change in the FIT group.

With respect to blood profile, there were no significant differences between the initial and final measurements for the FIT group, but the GRIT group showed reductions in both fasting blood glucose and triglyceride concentration (Figure 2 and Figure 3). The participants who completed the high intensity interval training decreased their blood glucose from $95.1 \mathrm{ml} / \mathrm{dL}$ to $92.1 \mathrm{ml} / \mathrm{dL}(-3.0 \%, \mathrm{p}<0.05)$ while their triglyceride concentration decreased from $116.4 \mathrm{ml} / \mathrm{dL}$ to $99.5 \mathrm{ml} / \mathrm{dL}(-16.9 \%$, $\mathrm{p}<0.05)$.

There were also numerous statistically significant differences between the initial and final strength and fitness measurements of the GRIT group that exceeded the results of the FIT group (Table 2, Figure 4). For instance, on average the GRIT participants increased their maximum number of push-ups in a minute from 37.4 to 44.8 , yielding a $19.8 \%$ increase $(\mathrm{p}<0.0001)$. These same participants demonstrated a $21.9 \%$ increase in back strength with an isometric deadlift improvement from $74.6 \mathrm{~kg}$ to $90.9 \mathrm{~kg}(\mathrm{p}<0.05)$. Finally, cardiovascular fitness improved by $6.4 \%$ in the GRIT group with an increase in estimated $\mathrm{VO}_{2}$ max of $2.8 \mathrm{ml} / \mathrm{kg} / \mathrm{min}(\mathrm{p}=0.05)$.

\section{Discussion}

The current ACSM physical activity guidelines suggest that moderate activity can be substituted or replaced with vigorous activity. The current study is one of the first to demonstrate the beneficial effects of high intensity training in individuals who meet the moderate activity guidelines. To add, the volume of exercise in our intervention groups was equal, which amplifies the findings on how intensity influences health and fitness. Finally, the current study also enhances the generalizability of the methods in that we utilized group fitness as the exercise model. The participants selected the cardiovascular classes that fit their schedule as well as their individual preference whereas previous studies utilized a treadmill or a cycle ergometer. These past methods were also completed in a controlled laboratory setting. In short, our protocol aids in refining the exercise prescription, demonstrates the benefits of high intensity interval training in a healthy population and utilizes methods that can be performed outside a research environment.

In support of our hypothesis, the six-week intervention successfully influenced numerous variables related to cardiovascular disease and are comparable to numerous studies referenced in a recent meta-analysis by Kessler 


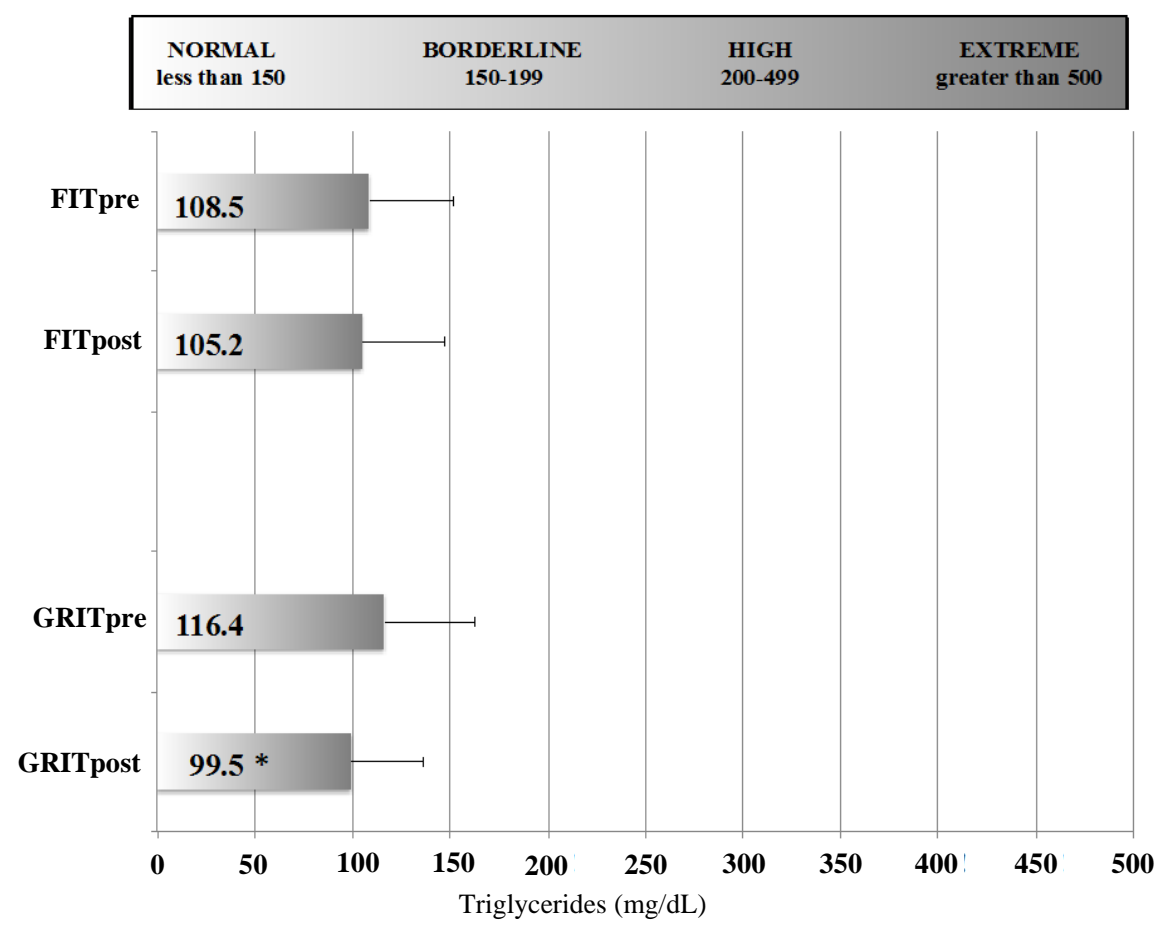

Figure 2. Mean \pm standard deviation for the initial and final measurements of triglycerides for the FIT and GRIT groups. The asterisk indicates a significant difference ( $\mathrm{p}$ 0.05 ) between the initial and final measurements. The bar at the top of the figure illustrates the category and range for each variable.

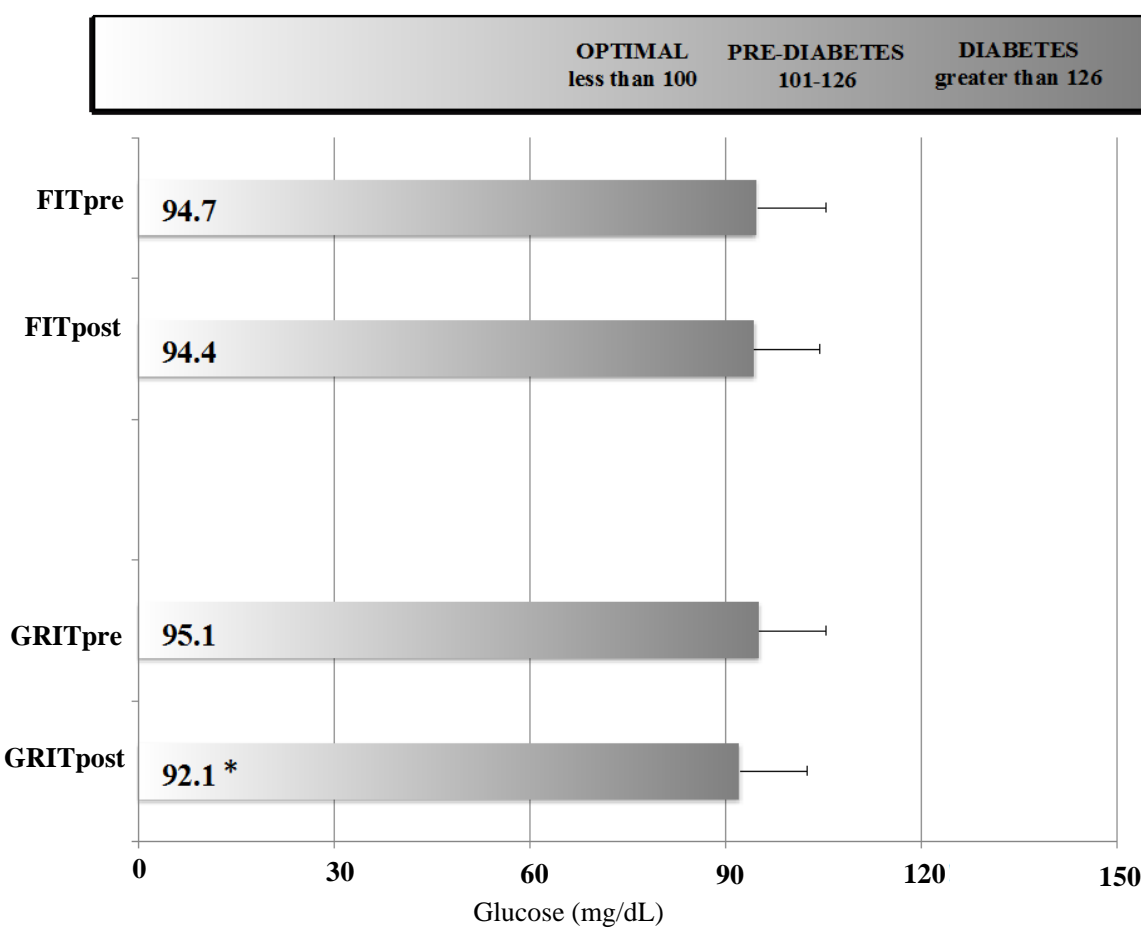

Figure 3. Mean \pm standard deviation for the initial and final measurements of glucose for the FIT and GRIT groups. The asterisk indicates a significant difference $(p<0.05)$ between the initial and final measurements of the individual group. The bar at the top of the figure illustrates the category and range for each variable. 
Table 2. Strength variables; mean (standard deviation).

\begin{tabular}{ccccc}
\hline & FIT initial & FIT final & GRIT initial & GRIT final \\
\hline Leg (kg) & $79.9(39.5)$ & $84.4(41.9)$ & $77.3(38.6)$ & $89.4(44.6)$ \\
Back (kg) & $77.8(36.6)$ & $84.3(39.5)$ & $74.6(35.3)$ & $90.9(43.5)$ \\
Upper Body (kg) & $35.7(8.8)$ & $38.2(7.4)$ & $37.4(7.6)$ & $44.8(8.7)$ \\
\hline
\end{tabular}

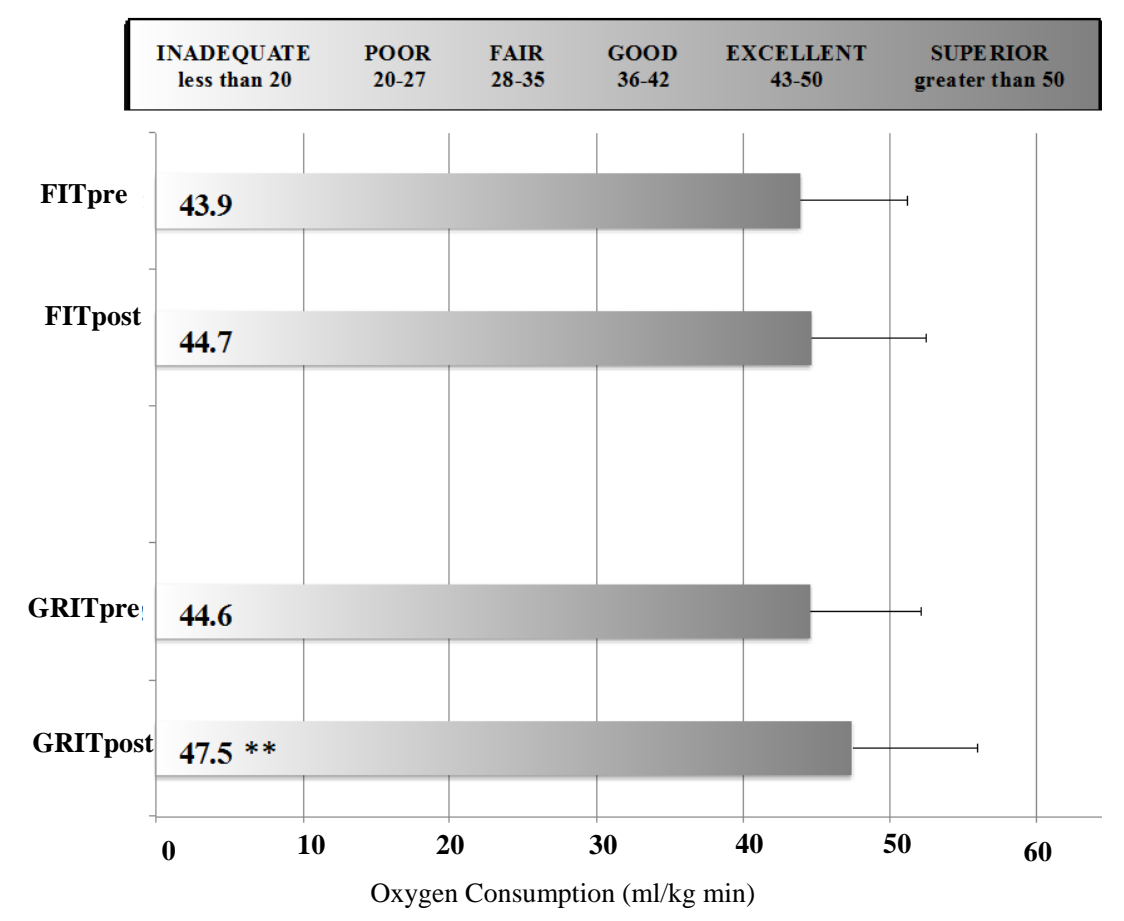

Figure 4. Mean \pm standard deviation for the initial and final measurements of oxygen consumption for the FIT and GRIT groups. The double asterisk indicates a significant difference ( $p<0.01$ ) between measurements of the individual group. The bar at the top of the figure illustrates the category and range for each variable.

et al. [11]. As a first example, Burgomaster et al. [12] and Macpherson et al. [13] each conducted 6-week intervention studies with young, untrained adults and compared sprint training to moderate intensity training on a cycle ergometer and a treadmill respectively. They reported improvements in oxygen consumption between 7.3\% - 11.5\% with sprint training. Our trained GRIT participants gained $6.4 \%$ in cardiovascular fitness with the addition of high intensity interval training. Second, Macpherson et al. [13] also found a significant reduction in fat mass with the interval training yielding a $4 \%$ greater reduction than continuous training. Likewise the participants who completed the GRIT protocol demonstrated a $2 \%$ decrease in body fat. Finally, similar to past studies with a duration of 6-week or less, there was no significant change in blood cholesterol levels. We did not find a significant reduction in total cholesterol or LDL-C levels nor did we find a significant enhancement of HDL-C concentrations. This opposes our hypothesis but is in parallel to previous studies of similar duration. Kessler et al. [11] recognized that vigorous intensity interventions with a duration of 8-weeks or greater reported significant changes in these cholesterol measures. In summary, the majority of our current results mimics previous literature results and demonstrates the unique benefits of vigorous training in an active participant population.

Interestingly, in contrast to referenced studies, but in agreement with our hypothesis, our 6-week protocol caused a clinically significant near $17 \%$ reduction in triglycerides despite the fact that the baseline values were not above normal. Moreira et al. [14] documented a similar magnitude of triglyceride decrease with a 12-week cycle ergometer protocol but the participants began the study with an elevated baseline level. It is possible that the LES MILLS GRIT ${ }^{\mathrm{TM}}$ series sessions from our study elicited the necessary caloric output expenditure war- 
ranted to cause hypotriglyceridemia [15]. One distinguishing feature of our intervention methodology was the inclusion of strength training in the LES MILLS GRITTM classes. The participants completed dynamic, integrated full body exercises such as clean and presses, deadlifts, dead rows, upright rows, and hang cleans with loaded bars. Perhaps interval training with weights provokes a greater change in triglyceride level, which would be a novel focus for a future study.

The incorporation of moderate intensity strength exercises in the FIT and GRIT protocols as well as the high intensity strength exercises in the GRIT protocol prompted us to measure upper body, back, and leg strength. These variables are rare, if ever, incorporated into past research since previous methodology is typically performed on a treadmill or cycle ergometer where strength gains would not be assumed. We documented significant upper body and back strength gains. The FIT and GRIT protocols utilized similar exercises such as push-up and clean and press variations, however the interval based protocol possibly enabled the participants to extend their range of motion and lift larger mass due to the rest periods, which led to the significant increases in strength.

These results illustrate a novel finding that individuals who already participate in the recommended physical activity guidelines can improve their health, fitness and strength with the addition of 2, 30-minute sessions of high intensity interval training per week. Our intervention was effective in reducing body fat mass and triglyceride concentration and enhancing lean body mass, glucose tolerance, cardiovascular fitness, and strength.

\section{Acknowledgements}

We thank The Athletic Club of State College for providing complimentary memberships. We also want to recognize the dedicated participants, their work created these impressive results. Finally, the study would not be possible without the elite North Club instructor team and the dedicated Pennsylvania State University kinesiology student assistants.

\section{Conflict of Interest}

While Les Mills International was supportive of the present study they did not have access to the data for analyses.

\section{References}

[1] Roger, V.L., Go, A.S., Lloyd-Jones, D.M., et al. (2011) Heart Disease and Stroke Statistics—2012 Update: A Report from the American Heart Association. Circulation, 125, e2-e220.

[2] Morrow Jr., J.R., Jackson, A.W., Bazzarre, T.L., et al. (1999) A One-Year Follow-Up to Physical Activity and Health. A Report of the Surgeon General. American Journal of Preventive Medicine, 17, 24-30. http://dx.doi.org/10.1016/S0749-3797(99)00030-6

[3] Garber, C.E., Blissmer, B., Deschenes, M.R., et al. (2011) American College of Sports Medicine Position Stand. Quantity and Quality of Exercise for Developing and Maintaining Cardiorespiratory, Musculoskeletal, and Neuromotor Fitness in Apparently Healthy Adults: Guidance for Prescribing Exercise. Medicine \& Science in Sports \& Exercise, 43, 1334-1359. http://dx.doi.org/10.1249/MSS.0b013e318213fefb

[4] Shephard, R.J. (2001) Absolute versus Relative Intensity of Physical Activity in a Dose-Response Context. Medicine \& Science in Sports \& Exercise, 33, S400-S418. http://dx.doi.org/10.1097/00005768-200106001-00008

[5] Swain, D.P. and Franklin, B.A. (2006) Comparison of Cardioprotective Benefits of Vigorous versus Moderate Intensity Aerobic Exercise. American Journal of Cardiology, 97, 141-147. http://dx.doi.org/10.1016/j.amjcard.2005.07.130

[6] O’Donovan, G., Owen, A., Bird, S.R., et al. (2005) Changes in Cardiorespiratory Fitness and Coronary Heart Disease Risk Factors Following 24 wk of Moderate- or High-Intensity Exercise of Equal Energy Cost. Journal of Applied Physiology, 98, 1619-1625. http://dx.doi.org/10.1152/japplphysiol.01310.2004

[7] Kraus, W.E., Houmard, J.A., Duscha, B.D., et al. (2002) Effects of the Amount and Intensity of Exercise on Plasma Lipoproteins. New England Journal of Medicine, 347, 1483-1492. http://dx.doi.org/10.1056/NEJMoa020194

[8] Cornelissen, V.A., Arnout, J., Holvoet, P., et al. (2009) Influence of Exercise at Lower and Higher Intensity on Blood Pressure and Cardiovascular Risk Factors at Older Age. Journal of Hypertension, 27, 753-762. http://dx.doi.org/10.1097/HJH.0b013e328322cf60

[9] Nybo, L., Sundstrup, E., Jakobsen, M.D., et al. (2010) High-Intensity Training versus Traditional Exercise Interventions for Promoting Health. Medicine \& Science in Sports \& Exercise, 42, 1951-1958. 
http://dx.doi.org/10.1249/MSS.0b013e3181d99203

[10] DiPietro, L., Dziura, J., Yeckel, C.W. and Neufer, P.D. (2006) Exercise and Improved Insulin Sensitivity in Older Women: Evidence of the Enduring Benefits of Higher Intensity Training. Journal of Applied Physiology, 100, 142-149. http://dx.doi.org/10.1152/japplphysiol.00474.2005

[11] Kessler, H.S., Sisson, S.B. and Short, K.R. (2012) The Potential for High-Intensity Interval Training to Reduce Cardiometabolic Disease Risk. Sports Medicine, 42, 489-509. http://dx.doi.org/10.2165/11630910-000000000-00000

[12] Burgomaster, K.A., Howarth, K.R., Philips, S.M., et al. (2008) Similar Metabolic Adaptations during Exercise after Low Volume Sprint Interval and Traditional Endurance Training in Humans. Journal of Physiology, 586, 151-160. http://dx.doi.org/10.1113/jphysiol.2007.142109

[13] Macpherson, R.E., Hazell, T.J., Olver, T.D., et al. (2011) Run Sprint Interval Training Improves Aerobic Performance but Not Maximal Cardiac Output. Medicine \& Science in Sports \& Exercise, 43, 115-122. http://dx.doi.org/10.1249/MSS.0b013e3181e5eacd

[14] Moreira, M.M., Souza, H.P., Schwingel, P.A., et al. (2008) Effects of Aerobic and Anaerobic Exercise on Cardiac Risk Variables in Overweight Adults. Arquivos Brasileiros de Cardiologia, 91, 200-206.

[15] Bellou, E., Magkos, F., Kouka, R., et al. (2013) Effect of High-Intensity Interval Exercise on Basal Triglyceride Metabolism in Non-Obese Men. Applied Physiology, Nutrition, and Metabolism, 38, 823-829. http://dx.doi.org/10.1139/apnm-2012-0468 